\title{
79 On the Upper Limit of the Frequency of the Transversal Vibration of Prismatic Bars.
}

\author{
By Kyoji SUYeHIRo, M.I.A. \\ Earthquake Research Institute, Tokyo Imperial University.
}

(Comm. June 12th, 1928.)

In dealing with the vibration of machine parts and structures, the study of which has, of late, become an important branch of the science of engineering, the resistance against the vibration is very seldom, if ever, considered; thereby in some cases one might come to a false conclusion.

In the present paper the author shall treat of the transversal vibration of prismatic bars subjected to resistance; this problem may have an important bearing on applied seismology and also to a certain measure on acoustics. The resistance is evidently composed of two sorts, one of them being an air resistance and the other an internal resistance due to solid viscosity. The fact that the former is insignificant compared with the latter was shown by Lord Kelvin" ${ }^{1)}$ and other investigators. Therefore, the air resistance will not be taken into consideration.

The solid viscosity was first studied by $W$. Voigt and recently by $\mathrm{K}$. Honda. ${ }^{2}$ According to these investigators, in analogy to the viscosity of fluid, the viscosity of solid is classed into two kinds :-

the tangential viscosity, $\quad \eta \frac{\partial \phi}{\partial t}$
and the normal viscosity, $\quad \xi \frac{\partial e}{\partial t}$

in which $\phi$ and $e$ are shearing and tensile strains respectively. These investigators' view will be taken up.

To simplify the problem, let us take that the bar is uniform and straight, and has a longitudinal plane of symmetry, and the transversal vibration takes place parallel to this plane. Take the axis of the bar in the position of equilbrium as $x$, and an axis perpendicular to it and parallel to the plane of symmetry as $y$.

Then the equation of the lateral motion of an elementary slice having the thickness $\delta x$ is

1) Lord Kelvin's Mathematical and Physical Papers, Vol. III, p. 23.

2) K. Honda, Phil. Mag. Vol. XLII (1921), p. 115-123. 


$$
\rho A \frac{\partial^{2} y}{\partial t^{2}} \delta x=\frac{\partial F}{\partial x} \delta x+\frac{\eta}{\mu} \frac{\partial^{2} F}{\partial x \partial t} \delta x
$$

in which $A$ is the sectional area of the bar, $\rho$ its density, $\mu$ the modulus of rigidity and $F$ the shearing force at the section.

Again, if we denote the moment of inertia of the cross section about its neutral axis by $I$, and the Young's modulus by $E$, the equation of the angular motion of the elementary slice is

$$
\rho I \frac{\partial^{2}}{\partial t^{2}}\left(\frac{\partial y}{\partial x}\right) \delta x=-\frac{\partial M}{\partial x} \partial x+F \partial x-\frac{\xi}{E} \frac{\partial^{2} M}{\partial x \partial t} \delta x+\frac{\eta}{\mu} \frac{\partial F}{\partial t} \hat{\partial} x \ldots
$$

Taking off $\delta x$ from (1) and (2), and eliminating $F$ between them, we have

$$
\rho A \frac{\partial^{2} y}{\partial t^{2}}=\rho I \frac{\partial^{2}}{\partial t^{2}}\left(\frac{\partial^{2} y}{\partial x^{2}}\right)+\frac{\partial^{2} M}{\partial x^{2}}+\frac{\xi}{E} \frac{\partial^{3} M}{\partial x^{2} \partial t}
$$

Remembering that $M=-E I \frac{\partial^{2} y}{\partial x^{2}}$ we obtain

$$
\rho A \frac{\partial^{2} y}{\partial t^{2}}=\rho I \frac{\partial^{2}}{\partial t^{2}}\left(\frac{\partial^{2} y}{\partial x^{2}}\right)-E I \frac{\partial^{4} y}{\partial x^{4}}-\xi I \frac{\partial^{5} y}{\partial x^{4} \partial t}
$$

If we suppose that the transverse dimensions of the bar are small compared with its length, and confine our attention to the waves having the lengths sufficiently larger than the transverse dimensions, the first term of the right-hand member may be neglected. Then the equation of motion for the resisted vibration of bars takes the form,

$$
\rho A \frac{\partial^{2} y}{\partial t^{2}}=-E I \frac{\partial^{4} y}{\partial x^{4}}-\xi I \frac{\partial^{5} y}{\partial x^{4} \partial t}
$$

This equation has already been obtained by Sezawa ${ }^{1)}$ and needs not recapitulating. But as he did neither mention the process of obtaining it nor show the physical meaning of $\xi$, the author described the procedure in order to show the physical meaning of $\xi$ and also to point out the extent of the applicability of the equation (4).

Now it is well known that the motion expressed by an equation as (4) may be analysed into component motions corresponding to the variation of normal co-ordinate. Accordingly put

$$
y=u_{r} \phi_{r}
$$

in which $u_{r}$ is a normal function and $\phi_{r}$ the corresponding normal co-ordinate. Substituting this into (4), we obtain

1) K. Sezawa, Bull. Earthq. Res. Inst. Vol. III (1927), p. 50. 


$$
\frac{d^{4} u_{r}}{d x^{4}}-m_{r}^{4} u_{r}=0
$$

and

$$
\frac{d^{2} \phi_{r}}{d t^{2}}+m_{r}^{4} \frac{\xi}{\rho} x^{2} \frac{d \phi_{r}}{d t}+m_{r}^{4} \frac{E}{\rho} x^{2} \phi_{r}=0
$$

where $m_{r}=\frac{n_{r}}{l}, n_{r}$ being the number characterizing the $r^{\text {th }}$ order of vibration and $l$ the length of the bar, and $x^{2}$ stands for $I / A$.

From the latter equation we have

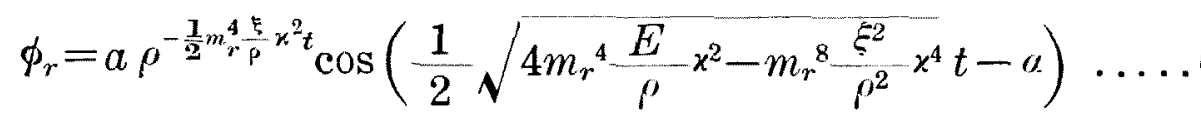

This expression tells that the transverse vibration of a bar is impossible unless

$$
m_{r}{ }^{8} \frac{\xi^{2}}{\rho^{2}} x^{4}<4 m_{r}{ }^{4} \frac{E}{\rho} x^{2}
$$

or

$$
\frac{\pi \xi}{E}<\frac{2 \pi}{m_{r}^{2} \chi} \sqrt{\frac{\rho}{E}}
$$

which can be written

$$
\frac{\pi \xi}{E}<T_{r}
$$

$T_{r}$ being the period of the vibration corresponding to $r$.

For a steel bar $\xi=5 \times 10^{8}$ (the value given by Honda) and $E=2 \times 10^{12}$. Therefore, $\frac{\xi}{E}=2.5 \times 10^{-4}$, and $\frac{\pi \xi}{E} \fallingdotseq \frac{1}{1,000} \mathrm{sec}$.

Thus, if the value of $\xi$ given by Honda is correct, the frequency of the purely transversal vibration of a steel bar cannot exceed 1,000 times per sec. ; such a deduction cannot be justisfied by our experience.

Next, with regard to the damped vibration of bars built of ordinary building materials, as their viscous property has not been much studied yet, it cannot be treated of exactly. The following deduction may, however, serves to give us some rough idea about it.

The intensity of the resistance against the transversal vibration may be found from the exponential term in (5),

$$
\rho^{-\frac{1}{2} m_{r}^{4} \frac{5}{\rho} x^{2} t}=\rho^{-\frac{1}{2} k t}
$$

in which $k$ stands for $\frac{\xi}{E} \frac{4 \pi^{2}}{T_{r}{ }^{2}}$. Therefore, if the logarithmic decre- 
ment of the amplitude and the natural period of vibration are given, we can guess the value of $\frac{\xi}{E}$. Making use of Omori's experiments on brick pillars, we arrive at the following results :

1) A brick column, ${ }^{11} 4.95 \mathrm{~m}$. high $\times 0.455 \mathrm{~m}$. broad $\times 0.225 \mathrm{~m}$. deep Period of fundamental vibration $=0.26 \mathrm{sec}$. Logarithmic decrement $\quad=0.148$

From these data, we obtain

$$
\frac{\xi}{E}=1.95 \times 10^{-3}
$$

Therefore, by (6)

$$
\mathrm{T}>\frac{1}{160} \mathrm{sec}
$$

Thus in this case the frequency cannot exceed 160 times per sec.

If we assume that the ratio of the frequencies of the vibrations of different orders is the same as that of an ideal cantilever, this upper limit of frequency is less than that of the 5th order.

2) A brick chimney, ${ }^{2)} 15.45 \mathrm{~m}$. high $\times 1.32 \mathrm{~m}$. square.

Period of fundamental vibration $=1.03 \mathrm{sec}$.

Logarithmic decrement $\quad=0.06$

From these data, we get

$$
\frac{\xi}{E}=3.13 \times 10^{-3}
$$

Therefore

$$
T>\frac{1}{100} \text { sec. }
$$

Under the same assumption as above, this frequency is less than that of the 7 th order.

It should be kept in mind that in both cases some portion of the energy of vibration might have dissipated through the fixed end. If so, the figures given above may, under circumstances, have not much value.

1) F. Omori, Bull, Imp. Earthq. Inv. Com. Vol. II No. 3 (1908), p. 224.

2) F. Omori, Pub. Imp. Earthq. Inv. Com. No. 12 (1902) p. 29. 